Notfall Rettungsmed 2013 · 16:89-91

DOI 10.1007/s10049-012-1643-0

Online publiziert: 7. März 2013

c) Springer-Verlag Berlin Heidelberg 2013

U. Kreimeier ${ }^{1}$ H.-R. Arntz ${ }^{2}$

${ }^{1}$ Klinik für Anaesthesiologie, Klinikum der Universität München

2 Medizinische KInik II, Kardiologie und Pulmologie,

Charité - Universitätsmedizin Berlin, Campus Benjamin Franklin, Berlin

\title{
Gesetz, Verordnung, Ausführungs- verordnung, Richtlinie, Empfehlung, Vereinbarung, Leitlinie
}

\section{Feine Unterschiede!}

Für die Notfallversorgung der Bevölkerung sind gestaffelte Hilfeleistungssysteme erforderlich. Nicht lebensbedrohliche Erkrankungen und Verletzungen werden durch Vertragsärzte und Notfallpraxen versorgt. Lebensbedrohliche Notfälle sind Aufgabe des Notarztes und klinischer Versorgungsstrukturen. Diese Versorgungsstrukturen müssen aufeinander abgestimmt werden [1]. Hierzu bedarf es bestimmter Voraussetzungen, zu denen neben Personal, Ausstattung des Rettungsmittels und Einsatzkonzept übergeordnete Vorgaben gehören.

Die Länder haben das Recht zur Gesetzgebung, soweit dieses Grundgesetz nicht dem Bund Gesetzgebungsbefugnisse verleiht. (Artikel 70, Absatz 1 Grundgesetz der Bundesrepublik Deutschland).

Ausgehend von der Prämisse der orginären Länderzuständigkeit hat das Grundgesetz dem Bund überwiegend die Kompetenzen im Bereich der Gesetzgebung übertragen. In der Zuständigkeit der Länder verbleiben wenige Bereiche, wie z. B. die Bildung, die Kultur und das Polizei- und Ordnungsrecht.

Der weit überwiegende Teil der Gesetze wird vom Deutschen Bundestag und damit vom Bund beschlossen. Die Länder wirken jedoch über den Bundesrat an der Gesetzgebung des Bundes mit [3]. Von der Volksvertretung beschlossen, sind Gestze für die Adressaten verbindlich.

\section{Verordnungen}

Verordnungen sind Rechtsnormen, die von Exekutivorganen erlassen werden.
Sie verweisen auf Gesetze. In den deutschsprachigen Ländern ist eine Verordnung eine Rechtsnorm, die in der Regel durch eine Regierung oder Verwaltungsstelle (Behörde) erlassen wird. Die Voraussetzungen für den Erlass einer Verordnung und der Umfang dessen, was eine Verordnung zulässigerweise regeln darf, unterscheiden sich zwischen Deutschland, Österreich und der Schweiz [7].

Ein parlamentarisches Gesetzgebungsverfahren dauert fast immer mehrere Monate - manchmal noch länger -, während Verordnungen in der Regel etwas schneller erlassen werden können. Deswegen ist es in vielen Bereichen gängige Praxis, dass der Gesetzgeber Details v. a. technischer Art und solche des Verwaltungsvollzugs nicht selbst regelt, sondern die Verwaltung ermächtigt, dies in einer Rechtsverordnung zu tun [7].

Eine der bekanntesten Ausführungsverordnungen eines Gesetzes im Bereich der Rettungsdienstes ist die „Verordnung zur Ausführung des Bayerischen Rettungsdienstgesetzes (AVBayRDG)“ vom 30.11.2010 [2]. In 4 Verordnungsteilen werden Organisation und Durchführung, Finanzierung des öffentlichen Rettungsdienstes, Schiedsstellen sowie Übergangsund Schlussbestimmungen dargelegt.

\section{Richtlinien, Empfehlungen, Vereinbarungen}

Eine Richtlinie ist eine Handlungsvorschrift mit bindendem Charakter, aber kein förmliches Gesetz. Es gibt technische Richtlinien, die von einer Organisa- tion ausgegeben werden und einen Standard vorgeben, der den Stand der Technik widerspiegelt. Im Gegensatz hierzu werden rechtliche Richtlinien von einem dazu formell gesetzlich ermächtigten Gremium beschlossen. In beiden Fällen haben Richtlinien einen bestimmten Geltungsbereich, der je nach dem Anwendungsfall z. B. arbeitsrechtlich auch sanktionierbar ist [6].

Empfehlungen werden zumeist von Fachgesellschaften oder berufsständischen Interessensvertretungen bzw. Fachgruppen herausgegeben und bieten eine Orientierung im beruflichen Alltag. In Form von Vereinbarungen kann z. B. die Zusammenarbeit mit anderen Berufsgruppen im Konsens festgelegt werden.

\section{Leitlinie und Evidenz in der Medizin}

Evidenzbasierte Medizin (EBM) bedeutet, Entscheidungen über einen zu erwartenden Nutzen einer medizinischen Methode vor dem Hintergrund der aktuellen wissenschaftlichen Erkenntnisse zu treffen. Hierfür werden wissenschaftliche Studien zu einer Fragstellung systematisch gesucht, bezüglich ihrer Qualität bewertet und die Ergebnisse hinsichtlich ihrer Übertragbarkeit auf das aktuelle Problem beurteilt [5]. Die evidenzbasierte Medizin hat im Gesundheitswesen enorme Resonanz gefunden, seit im Zusammenhang mit der Kostenexplosion erkannt wurde, dass die im internationalen Vergleich hohe Gesundheitsausgaben keineswegs regelhaft einen günstigen Effekt auf die Volksgesundheit zu haben scheinen. 
Juristisch stellen Leitlinien somit einen Behandlungskorridor dar, der einen bestimmten Freiraum nach entsprechender Begründung für die Wahl des Vorgehens einräumt. Medizinisch sind Leitlinien Vorgaben für das praktische Vorgehen. Eine der bekanntesten Leitlinien sind die „ERC-Leitlinien zur Kardiopulmonalen Reanimation 2010“, die in autorisierter deutschsprachiger Übersetzung durch den Deutschen Rat für Wiederbelebung - German Resuscitation Council (GRC) -, den Austrian Resuscitation Council (ARC) und den Swiss Resuscitation Council (SRC) im November 2010 in Notfall + Rettungsmedizin veröffentlicht wurden [4].

\section{Hauptproblem: Zeit und gesetzliche Vorgaben}

Alarmierungszeit, Ausrückzeit, Eintreffzeit, ... Zeitstempel sind ein wichtiges Element in der Ablaufstruktur eines notfallmedizinischen Einsatzes. Zeit ist aber auch ein physiologisches Moment, welches z. B. im Fall eines Kreislaufstillstands das (neurologische) Outcome bedingt. Ehe professionelle Retter am Notfallort eintreffen, sollten deshalb Laien mit der Reanimation beginnen.

Hier schließt sich der Kreis: Jeder ist verpflichtet $\mathrm{zu}$ helfen! Dies ist gesetzlich normiert. Nach $\$ 323 \mathrm{c}$ Strafgesetzbuch (StGB) wird derjenige, der bei einem Unglücksfall keine Hilfe leistet, obwohl dies erforderlich und möglich war, mit Freiheitsstrafe bis zu 1 Jahr oder mit Geldstrafe bestraft (unterlassene Hilfeleistung).

\section{„Das" Eckpunktepapier}

Häufig - wie auch eingangs dieses Editorials - wird die in Notfall + Rettungsmedizin erfolgte Veröffentlichung „Eckpunktepapier zur notfallmedizinischen Versorgung der Bevölkerung in Klinik und Präklinik“ [1] zitiert, wenn es um den Zeitablauf bis zur definitiven Therapie in der Notfallmedizin geht. Für die Tracer-Diagnosen ST-Hebungsinfarkt, Schlaganfall, Schädel-Hirn-Trauma und Schwerverletzte wurden von einer Expertengruppe strukturelle und Zeitvorgaben gemacht, um eine qualitativ hochwertige Akutversorgung zu ermöglichen. Wenn das Motto gilt „Time is brain“, wird z. B. beim Schlag- anfall gefordert, das innerhalb von $90 \mathrm{~min}$ die Entscheidung über die Lyse getroffen wird bzw. maximal 120 min bis zur Lyse vergehen dürfen.

Dieses Eckpunktepapier wurde von einer Reihe von Fachgesellschaften, Organisationen und Vereinigungen verabschiedet und demonstriert, welche große Bedeutung die Konsensfindung in der notfallmedizinischen Versorgung hat und welches Potenzial in einer Konsensbildung der Beteiligten in der notfallmedizinischen Versorgung liegt.

\section{Sehr geehrte Leserinnen und Leser,}

die Vereinheitlichung von Vorgehensweisen anhand von Leitlinien und Algorithmen hat sich in den letzten Jahren zu einem wichtigen Werkzeug bei der interdisziplinären und interprofessionellen $\mathrm{Zu}$ sammenarbeit entwickelt. In der Notfallmedizin besteht neben Ressourcenverknappung insbesondere ein Mangel an „Zeit“, damit die Rettungskette schnell, reibungslos und effektiv umgesetzt wird. Nutzen wir die uns zur Verfügung stehenden Ressourcen effizient, um neben den gesetzgeberischen Vorgaben konsentierte Handlungsvorgaben zu erarbeiten und umzusetzen und damit eine qualitativ hochwertige notfallmedizinische Versorgung der Bevölkerung zu gewährleisten.

Alles entsteht und vergeht nach Gesetz. Doch über des Menschen Leben, dem köstlichen Schatz, herrschet ein schwankendes Los. Johann Wolfgang von Goethe, Elegien: Euphrosyne.

Ihre

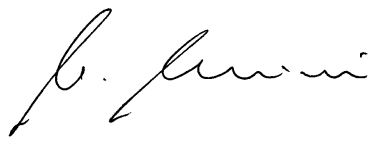

U. Kreimeier

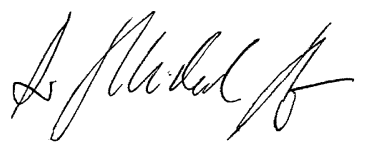

H.-R. Arntz

\section{Korrespondenzadresse}

Prof. Dr. U. Kreimeier

Klinik für Anaesthesiologie,

Klinikum der Universität München

Nussbaumstraße 20, 80336 München

Uwe.Kreimeier@med.uni-muenchen.de

\section{Compliance with Ethics Guidelines}

Conflict of interest. H.R. Arntz and U. Kreimeier declare that they have no conflict of interest.

This article does not contain any studies with human or animal subjects.

\section{Literatur}

1. Altemeyer FW, Altemeyer $\mathrm{KH}$, Blumenberg D et al. (2008) Eckpunktepapier zur notfallmedizinischen Versorgung der Bevölkerung in Klinik und Präklinik. Notfall Rettungsmed 11:421-422

2. Bayerische Staatsregierung. Verordnung zur Ausführung des Bayerischen Rettngsdienstgesetzes (AVBayRDG) vom 30.11.2010. http://www.verkuendung-bayern.de/gvbl/jahrgang:2010/heftnummer:21/seite:786. Online-Zugriff am 23.07.2012.

3. Bundesrat. Das Gesetzgebungsverfahren. http:// www.bundesrat.de/nn_9552/DE/struktur/gesetzgebung/gesetzgebung-node.html?_nnn=true. Online-Zugriff am 23. Juli 2012.

4. ERC-Leitlinien 2010. Kardiopulmonale Reanimation. Notfall Rettungsmed 7:504-744.

5. Kreimeier U, Dirks B, Wenzel V (2008) Evidenzbasierte Notfallmedizin: Perspektiven. Einklang von klinischer Erfahrung, wissenschaftlichen Erkenntnissen und EBM. Notfall Rettungsmed 11:18-24

6. Wikipedia. Richtlinie. http://de.wikipedia.org/wiki/ Richtlinie. Online-Zugriff am 23. Juli 2012

7. Wikipedia. Verordnung. http://de.wikipedia.org/ wiki/Verordnung. Online-Zugriff am 23. Juli 2012 


\section{In eigener Sache \\ Zur Einführung der Rubrik "Teamwork + Education"}

Einer der Erfolgsgaranten für erfolgreiche Organisationen ist Teamwork. Teamwork geht über die reine Zusammenarbeit hinaus: Es bezieht sich auf Techniken, Prozesse und Arbeitswerkzeuge ("tools"), um eine Gruppe von Individuen zu organisieren und, wichtiger noch, zu koordinieren, auf ein gemeinsames Ziel hinzuarbeiten. In der Medizin ist Teamwork direkt mit der Anwendung von Praktiken und Ressourcen verbunden: Aufbauend auf dem Gelernten, der Ausbildung und der Bereitschaft jedes Team-Mitglieds bedeutet dies, z.B. bei der Versorgung eines Patienten die bestmögliche Strategie einzusetzen, um eine effiziente und optimale Therapie zu ermöglichen. In einem Team müssen Verantwortlichkeiten festgelegt werden: Teamwork ist aber nur denkbar, wenn alle dasselbe wollen. Verschiedene Sichtweisen und Erfahrungen müssen somit vereint und auf eine gemeinsame Strategie gelenkt werden, und dies in der für die Notfallmedizin kritischen Zeitspanne.

Zu den Führungsaufgaben gehört auch, effektive Teams zu entwickeln. Mit der Tatsache, dass nicht jedes Teammitglied den gleichen beruflichen Ausbildungsweg hinter sich hat, sollte sich das Team um die Fähigkeiten des Einzelnen ergänzen. Dies schließt die Aus-, Fort- und Weiterbildung mit ein. Benötigt werden ebenfalls Strategien und Schlüsselelemente, die die Basis für das Wirken darstellen. Neben den Ausbildungs- und Weiterbildungskatalogen gehören hierzu die intrinsische Motivation, stetig dazulernen zu wollen („life-long learning"). Nichts in der medizinischen Therapie ist immerwährend, auch Leitlinien sind nicht in Stein gemeißelt, d.h. auch sie müssen in regelmäßigen Abständen auf Eignung und Korrektheit überprüft und ggf. angepasst werden.

Mit der neuen Rubrik „Teamwork + Education" werden wir in jeder Ausgabe Aspekte beleuchten, die den konzeptionellen Ansatz der Optimierung der Zusammenarbeit von Teams unterstützen.

\section{H.-R. Arntz \\ U. Kreimeier}

Editors-in-Chief

Leitthemenübersicht

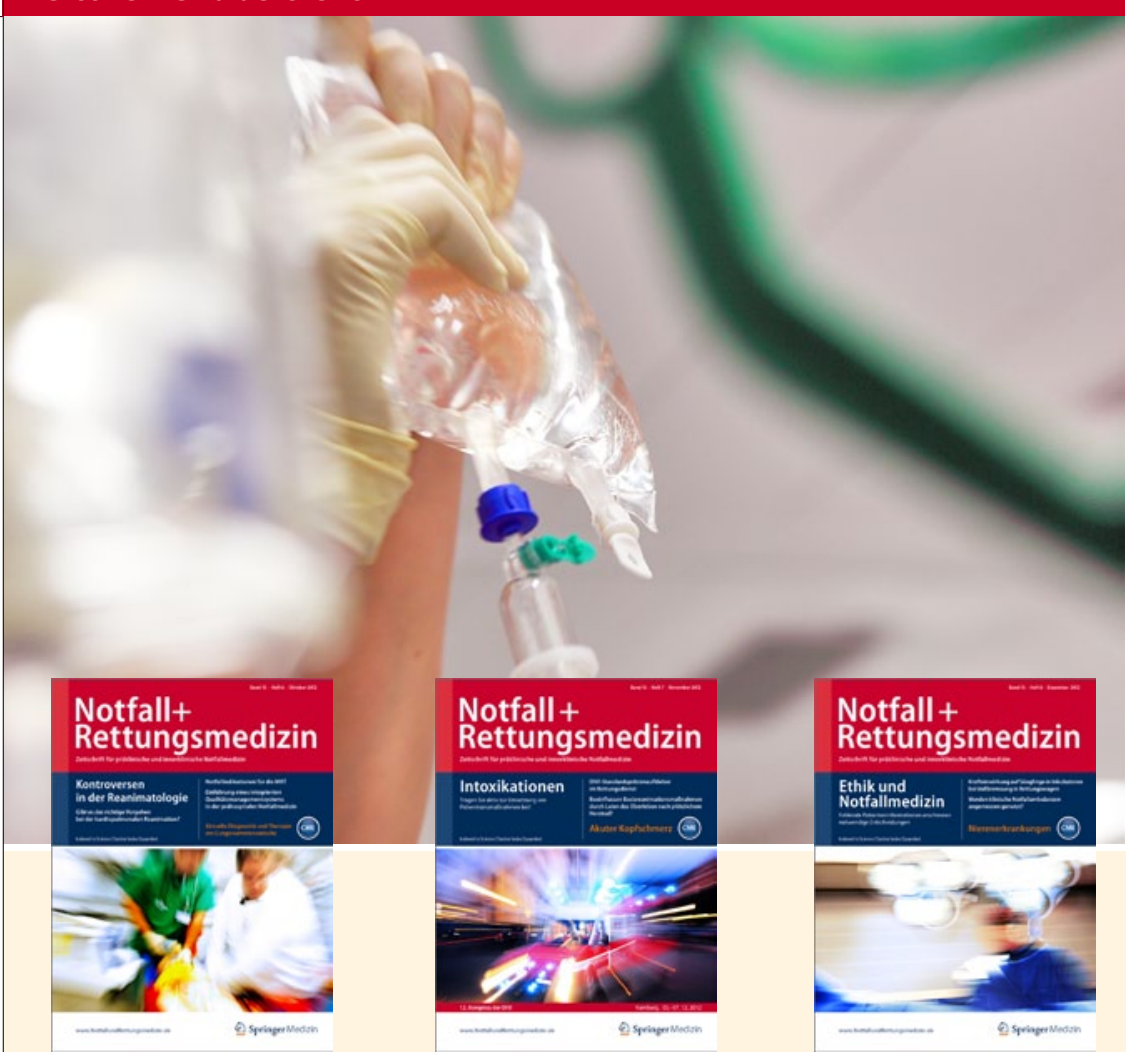

Die Notfall + Rettungsmedizin bietet Ihnen jeden Monat umfassende und aktuelle Beiträge zu interessanten Themenschwerpunkten aus allen Bereichen der Notfallmedizin. Möchten Sie ein bereits erschienenes Heft nachbestellen? Einzelne Ausgaben können Sie direkt bei unserem Kundenservice zum Preis von je EUR 35,-zzgl. Versandkosten beziehen:

\section{Überblick 2011}

1/11 Präklinisches Atemwegsmanagement

2/11 Lebensgefährliche Herzrhythmusstörungen

3/11 Notfallmedizin und Informationstechnologie

4/11 Leichte Schädel-Hirn-Traumata

5/11 Notaufnahme

6/11 Medizinrechtliche Aspekte der Notfallmedizin

7/11 Pädiatrische Notfälle

8/11 Advanced Technology in der Notfallmedizin

\section{Überblick 2012}

1/12 Risikomanagement in der Akutmedizin

2/12 Respiratiorsche Notfälle im Kindesalter

3/12 Weiterbildung in der Notfallmedizin

4/12 Schnittstellen in der Notfallmedizin

5/12 Notaufnahme: Arbeitsplatz mit Zukunft?

6/12 Kontroversen in der Reanimatologie

7/12 Intoxikationen

8/12 Ethik in der Notfallmedizin

So erreichen Sie unseren Kundenservice:

Springer Customer Service Center GmbH Kundenservice Zeitschriften

Haberstr. 7

69126 Heidelberg

Tel.: +496221345-4303

Fax: +496221 345-4229

E-Mail: leserservice@springer.com

\section{www.NotfallundRettungsmedizin.de}

